\title{
ESTÁGIO ATUAL DO CONHECIMENTO A CERCA DO MINÉRIO LATERÍTICO DE NÍQUEL NO BRASIL E NO MUNDO
}

\author{
Sonia Maria Barros de OLIVEIRA
}

\author{
RESUMO
}

\begin{abstract}
Este trabalho trata do "estado da arte" referente aos depósitos de níquel laterítico no Brasil e no mundo. São abordados aspectos referentes à características gerais dos depósitos e, principalmente, ao estudo das fases portadoras de níquel.
\end{abstract}

\section{ABSTRACT}

This paper deals with the "state of art" of nickeliferous deposits of lateritic origin in general and specially in Brazil. Geological characteristics of the deposits are discussed. Special attention is drawn to the evolution of knowledge on nickel-bearing phases.

\section{INTRODUÇÃO}

O níquel é considerado um metal estratégico devido à sua grande importância como matéria-prima na indústria do aço, e devido também à distribuição de suas reservas no mundo, concentradas em alguns poucos países. Há apenas restritas possibilidades para a substituição do níquel, que é usado principalmente na produção de aços inoxidáveis e de ligas metálicas.

Dois tipos de depósito são responsáveis pela produção do níquel: os sulfetos magmáticos e as lateritas. Os primeiros formam-se por segregação de líquidos sulfetados imiscíveis na base da câmara magmática, a partir do resfriamento de magmas de natureza máfica a ultramáfica, e são principalmente de idade proterozóica. Um exemplo de depósito sulfetado é o de Sudbury, no Canadá, um dos maiores produtores de níquel do mundo. Os depósitos de níquel laterítico formam-se pelo intenso intemperismo de rochas ultramáficas, e são, em sua maior parte, de idade cenozóica. Ocorrem, principalmente, em área de clima tropical, como na Nova Caledônia, nas Filipinas e no Brasil. Outros tipos de depósito de níquel são os veios hidrotermais contendo sulfetos e os nódulos do fundo dos oceanos que, atualmente, não apresentam interesse econômico.
As reservas mundiais são da ordem de 97.106 toneladas de níquel contido. Em torno da metade está localizada na Nova Caledônia, e o restante encontra-se principalmente na Indonésia, Canadá, URSS, Cuba e Austrália. Cerca de $72 \%$ do total é do tipo laterítico (DEYOUNG JR. et al., 1985).

A produção do níquel foi de $630.10^{3}$ toneladas de níquel contido, em 1982, das quais $65 \%$ provieram de minério sulfetado. Mais de $80 \%$ da produção derivou apenas de oito países: URSS, Canadá, Austrália, Nova Caledônia, Indonésia, Cuba, África do Sul e Filipinas. (DEYOUNG JR. et al., 1985).

A preferência pela exploração das reservas sulfetadas é explicada pelo fato de que esse tipo de minério pode ser facilmente concentrado através de técnicas bem estabelecidas, enquanto que o minério laterítico necessita de processos mais complicados, de alto consumo de energia, para seu tratamento. Além disso, os teores de níquel no minério sulfetado são mais altos, e os subprodutos como cobre, cobalto e platinóides aumentam consideravelmente o valor desse tipo de minério. No entanto, como, de longe, a maior parte das reservas é do tipo laterítico, este será provavelmente o minério mais explorado no futuro. 


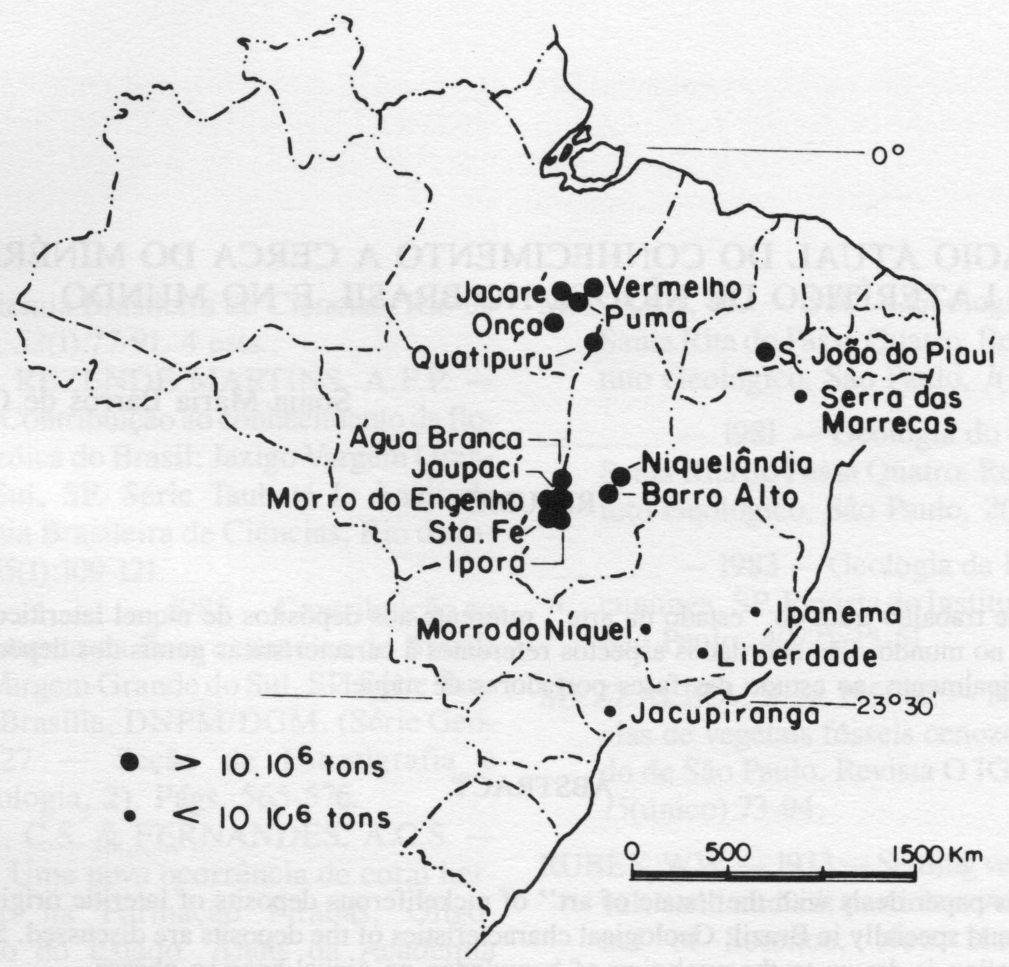

FIGURA 1 - Principais depósitos de níquel laterítico do Brasil.

\section{ASPECTOS ECONÔMICOS REFERENTES AO NÍQUEL NO BRASIL: HISTÓRICO E SITUAÇÃO ATUAL}

No Brasil, a primeira menção à ocorrência de minério de níquel data de 1889 , e é referente ao distrito de Jacupiranga, no Estado de São Paulo (FELICÍSSIMO, 1968). A partir de então, outras ocorrências foram mencionadas: Niquelândia (1906) em Goiás, Liberdade (1916) e Morro do Níquel (1922) em Minas Gerais, e Barro Alto (1934) em Goiás. Na segunda metade do século $\mathrm{XX}$, muitos depósitos foram descobertos, através da prospecção sistemática.

Hoje conkecem-se no Brasil importantes reservas de níquel, perfazendo $349,2.10^{6}$ toneladas de minério, com teores variando de 1,2 a 2,2\% Ni (SCHOBBENHAUS et al., 1984). Toda essa reserva provém de depósitos lateríticos, espalhados por todas as regiões do país, sendo apenas mais raros no Nordeste e no Sul. A figura 1 mostra os principais depósitos de níquel laterítico do Brasil, dando uma indicação sobre a dimensão de suas reservas.

Apenas dois depósitos de níquel sulfetado são conhecidos no Brasil: Americano do Brasil, em Goiás, com 5,0.106 toneladas e teores de $0,62 \% \mathrm{Ni}, 0,65 \% \mathrm{Cu}$ e $0,04 \%$ Co (NILSON et al., 1986), e Fortaleza de Minas, em Minas Gerais, com $5,1.10^{6}$ toneladas de minério, com $2,55 \% \mathrm{Ni}, 0,45 \% \mathrm{Cu}$ e $0,07 \%$ Co (CRUZ et al., 1986).
$\mathrm{O}$ níquel foi explorado em pequena escala em Jacupiranga, durante a Segunda Guerra, e em Liberdade, de 1927 a 1975. Atualmente, existem no Brasil três distritos produtores de níquel, cujo produto final é tanto o níquel eletrolítico, como a liga ferro-níquel; a matéria-prima é nos dois casos o minério laterítico. A Companhia Níquel Tocantins S/A, que explora o depósito de Niquelândia-Sul, é a única produtora de níquel eletrolítico no Brasil. A liga ferro-níquel é produzida pela CODEMIN e pela Companhia Morro do Níquel, a partir do minério das minas de Niquelândia-Norte e de Morro do Níquel, respectivamente. Em 1987 a produção total no Brasil foi de 1.308.655 toneladas de minério, com 22.092 toneladas de níquel contido (Anuário Mineral Brasileiro, 1988).

O Brasil exporta a liga ferro-níquel, mas importa produtos manufaturados de níquel. A importação decresceu significativamente nos últimos anos, desde que a usina de NiquelândiaSul começou a produzir níquel eletrolítico (1980).

\section{EVOLUÇÃO DOS CONHECIMENTOS RELATIVOS ÀS LATERITAS NIQUELÍFERAS}

Até meados do século XIX, eram conhecidos apenas minérios de níquel de origem endógena, de tipo sulfetado. Em 1863 Garnier descobriu, na Nova Caledônia, um mineral de 
níquel de tipo diferente: era um silicato hidratado de magnésio e níquel que, em 1874 , foi denominado de "garnierita". Os primeiros trabalhos publicados sobre esse tipo de minério atribuíram-lhe origem magmática, devido ao aspecto filoneano dos veios garnieríticos. Apenas em 1903, sua origem, a partir do intemperismo de certos tipos de rocha, foi reconhecida e, a partir daí, aceita sem mais polêmica (AVIAS, 1978). Os depósitos da Nova Caledônia foram, assim, o primeiro exemplo de minério laterítico de níquel do tipo silicatado descrito no mundo. Só em meados do século XX, análises mais detalhadas dos perfis lateríticos, reconhecendo a existència de mais um tipo de minério, o oxidado, foram publicadas, com relação aos perfis da Nova Caledônia (LACROIX, 1942; CHETELÂT, 1947), do Brasil (PECORA, 1944) e de Cuba (VLETTER, 1955).

O aumento da demanda de níquel no mundo de pós-guerra explica o crescente interesse pelo estudo dos perfis lateríticos niquelíferos. Em nível regional, vários depósitos foram descritos: Venezuela (JURKOVIC, 1963), Guiné (BONIFAS, 1959; PERCIVAL, 1965), Filipinas (SANTOS-YNIGO, 1964), Austrália (ZEISSINK, 1969), Brasil (COSTA, 1970), etc. Esses trabalhos caracterizaram os diferentes horizontes encontrados numa grande variedade de perfis.

Estudos mineralógicos de detalhe, com o objetivo de caracterizar as fases portadoras de níquel, foram empreendidos a partir dos anos sessenta. Com relação às garnieritas, os trabalhos de MAKSIMOVIC (1966, 1975), BRINDLEY \& HANG (1973), HANG \& BRINDLEY (1973), UYEDA et al. (1973), SPRINGER (1974), BRINDLEY \& MAKSIMOVIC (1974) e BRINDLEY (1978), por exemplo, indicaram que se trata de uma mistura de silicatos magnesianos e niquelíferos hidratados, com distâncias interplanares de 7A (minerais 1:1) e 10A (minerais 2:1), sendo as séries mais comuns a da lizardita-nepouita e a da kerolita-pimelita. Outros filossilicatos portadores de níquel foram descritos nos perfis lateríticos, como as cloritas, vermiculitas e esmectitas (MAKSIMOVIC, 1972; WIEWIORA \& SZPILA, 1975). E o níquel também se mostrou presente como compostos amorfos, acumulados em microfissuras ou na superfície das serpentinas (GONI \& GUILLEMIN, 1964; AMMOU CHOKROUM, 1972), ou ainda substituindo o magnésio no retículo cristalino desses minerais (COY-YLL \& GUILLOUX, 1972). No minério oxidado, o níquel evidenciou-se associado à goethita (COMBES,
1963) e aos hidróxidos de manganês e cobalto (AMMOU CHOKROUM, 1969).

Investigações experimentais, simulando o intemperismo das rochas ultramáficas no laboratório, foram realizadas por PEDRO \& BITTAR (1966) PEDRO \& DELMAS (1971), DELMAS (1972), BIBENT (1972), BIBENT \& BOURGEOIS (1973) e PERRUCHOT (1976a).

Esses estudos puseram em evidência a alteração da olivina por dissolução incongruente, com resíduo amorfo enriquecido em níquel, precedendo à da serpentina, esta por dissolução praticamente congruente. De modo geral, ficou demonstrada a lixiviação do Si e $\mathrm{Mg}$ e a acumulação residual do Fe. Nesses aspectos, a alteração in vitro reproduziu muito bem o que acontece na natureza. No entanto, no laboratório, em condições hidrolíticas, ocorre lixiviação preferencial do $\mathrm{Si}$ em relação ao $\mathrm{Mg}$, o que é o oposto do que se dá na natureza. Uma possível explicação para essa inversão estaria relacionada à natureza mais alcalina das soluções de alteração geradas nas condições de laboratório.

No sentido de interpretar os mecanismos formadores das acumulações de níquel laterítico ao nível de um depósito como um todo, foi fundamental a contribuição de TRESCASES (1975). Seu trabalho na Nova Caledônia, apresentando um balanço quantitativo do níquel nos vários setores da paisagem, esclareceu importantes aspectos concernentes ao ciclo desse elemento no ambiente supérgeno.

Três artigos de síntese sobre a gênese dos depósitos de níquel laterítico devem ser mencionados: LELONG et al. (1976), GOLIGHTLY (1981) e SCHELLMANN (1983). O primeiro trata dos mecanismos de acumulação do níquel nos perfis lateríticos e dos fatores que controlam essa acumulação. GOLIGHTLY (op.cit), além de relacionar a variação na estruturação dos perfis lateríticos com as condições climáticas e a composição da rocha parental, elabora um ensaio explicativo das paragêneses observadas, em termos das reações de equilíbrio dissoluçãoprecipitação de cada fase presente. Finalmente, no terceiro artigo, são detalhados os processos de formação do minério silicatado e oxidado.

Dos últimos anos da década de setenta em diante, os trabalhos publicados na literatura sobre o níquel laterítico estão ligados principalmente a dois temas (TRESCASES, 1986). O primeiro é o estudo de novos depósitos, ou o reestudo de depósitos já conhecidos: Nova Caledônia (TROLY et al., 1979), Filipinas (OGURA, 1977; MERCADO, 1981), Índia (SAHOO et al., 1981), Costa do Marfim (NAHON et al., 1982), Senegal (BLOT et al., 1976), Burma (SCHELLMANN, 1989), entre outros tantos, in- 
clusive os depósitos brasileiros, que serão tratados mais adiante. Nesses trabalhos, além de uma caracterização do perfil de alteração que permite evidenciar as filiações mineralógicas, alguns autores tentaram esclarecer os mecanismos de concentração do níquel na escala da paisagem. Assim, hoje se tem um quadro bastante satisfatório do papel dos fatores tectônicos, litológicos e morfoclimáticos no controle da gênese dos depósitos de níquel laterítico.

O segundo tema extensivamente tratado na literatura diz respeito a estudos de mais detalhe que se referem a aspectos cristaloquímicos das fases portadoras de níquel. O minério silicatado foi, neste aspecto, muito mais bem estudado que o minério oxidado. Dentre os trabalhos mais importantes referentes à cristaloquímica do níquel nos minerais do minério silicatado estão os de BISH \& BRINDLEY (1978), PONCELET et al. (1979), BRINDLEY et al. (1979), CERVELLE \& MAQUET (1982), GERARD \& HERBILLON (1983), PELLETIER (1983), MANCEAU \& CALAS (1983) e MANCEAU (1984). Mais recentemente, modelos experimentais do que acontece na natureza em relação às fases silicatadas encontradas em perfis de alteração de rochas ultramáficas têm sido propostos. Por exemplo, STROESSEL (1988) investigou aspectos termodinâmicos e cinéticos relativos à dissolução da kerolita em relação à sepiolita, e VELDE (1988) tratou de reproduzir no laboratório a formação de Ni-talco e Ni-serpentina em pseudomorfoses sobre diopsídio.

Com relação ao minério limonítico, podem ser mencionados os trabalhos de SCHELLMANN (1978), KUHNEL et al. (1978), MAQUET et al. (1981) e SCHWERTMANN \& LATHAN (1986). Nesses trabalhos há indicações de uma estreita associação entre óxidos de ferro e níquel, e são até apresentadas provas definitivas de substituição diadóquica eritre esses dois elementos (GERTH, 1990), a exemplo do que acontece com o Mn (STIERS \& SCHWERTMANN, 1985) e Co (SCHWERTMANN et al., 1989) nas goethitas sintéticas. Os asbolanos e outros óxidos de Mn portadores de Ni e Co vêm tendo seus aspectos cristaloquímicos elucidados, por exemplo, por CHUKHROV et al. (1983) e MANCEAU et al. (1987). A adsorção do níquel por produtos amorfos foi investigada experimentalmente por PERRUCHOT (1976b) e COUTY $\&$ PERRUCHOT (1980).

Esses estudos prosseguem e, fazendo uso de equipamento bastante sofisticado (espectroscopia de absorção no visível, infravermelho e raios $\mathrm{X}$, difração de raios $\mathrm{X}$ em microamostras, microscopia eletrônica de alta resolução e microssonda eletrônica, por exemplo), vêm abordando o problema da localização do níquel a escalas cada vez menores. Além de importante contribuição teórica, investigações dessa natureza trazem valiosa informação para orientar o desenvolvimento de processos no tratamento do minério.

\section{O CONHECIMENTO CIENTÍFICO A RESPEITO DAS LATERITAS NIQUELÍFERAS DO BRASIL}

No Brasil, embora depósitos de níquel laterítico fossem conhecidos desde o século passado, até meados da década de setenta só se encontravam na literatura estudos de caráter muito geral, abordando principalmente aspectos econômicos. Entre os mais recentes, estão os de FERRAN (1974), LAGES et al. (1976) e BERBERT (1977), por exemplo. A caracterização mineralógica e geoquímica dos perfis mineralizados começou a partir dessa época, e foi realizada, em sua maior parte, por um grupo de pesquisadores em geoquímica da alteração intempérica, pertencentes ao Departamento de Geologia Geral do Instituto de Geociências da Universidade de São Paulo. Os principais trabalhos produzidos por esse grupo e alguns colaboradores referem-se aos depósitos de Morro do Níquel (TRESCASES \& OLIVEIRA, 1978), Santa Fé (OLIVEIRA \& TRESCASES, 1980; OLIVEIRA, 1980a e b), Barro Alto (MELFI, 1974; TRESCASES \& OLIVEIRA, 1981), Niquelândia (COLIN, 1985; COLIN et al., 1985; OLIVEIRA \& TRESCASES, 1982), Vermelho (BERNARDELLI et al., 1983)', São João do Piauí (TRESCASES et al., 1986), Ipanema (ANGELI et al., 1984) e Jacupiranga (OLIVEIRA \& TRESCASES, 1985; OLIVEIRA et al., 1988). Uma síntese preliminar que deu conta das principais características de alguns depósitos brasileiros e propôs uma hipótese sobre sua gênese foi elaborada por MELFI et al. (1980). No quadro mais amplo das jazidas lateríticas do Brasil, alguns depósitos brasileiros são descritos por MELFI et al. (1988). Outros trabalhos que ajudaram a caracterizar os principais depósitos de níquel laterítico brasileiros podem ser citados: LANGER (1969) para Morro do Níquel, STACHE (1974) para Barro Alto, SANTOS (1974) para São João do Piauí, BARBOUR (1978), SILVA (1980) e BARBOUR \& HIPÓLITO (1983) para Santa Fé, ESSON \& SANTOS (1978a) para Liberdade, CORREA et al. (1984) para o Vermelho e ANGELI (1989) para Ipanema. Em 1986 o Departamento Nacional da Produção Mineral lançou o segundo volume da série "Principais Depósitos Minerais do Brasil”, onde consta uma descrição geral dos maciços de $\mathrm{Ni}$ quelândia, Barro Alto, Morro do Engenho, São 
João do Piauí, Vermelho, Onça, Puma e Jacaré. Recentemente, OLIVEIRA (1990) apresentou como trabalho de livre-docência ao Instituto de Geociências da Universidade de São Paulo uma síntese atualizada do conhecimento sobre os depósitos de níquel laterítico do Brasil.

A investigação de aspectos mineralógicos de mais detalhe, referentes aos minérios de níquel lateríticos do Brasil, é bastante incipiente. Além de alguns trabalhos de caráter geral publicados na década de setenta, como BRINDLEY \& SOUZA (1975 a e b), BOSIO et al. (1975), SOUZA et al. (1978) e ESSON \& SANTOS (1978b) para as fases silicatadas enriquecidas em níquel, provenientes de Niquelândia, Morro do Níquel, Jacupiranga, Barro Alto e Liberdade, quase mais nada consta da literatura. Merece menção apenas o trabalho de Tese de Doutoramento de COLIN (1985) sobre os produtos de alteração dos piroxenitos de Niquelândia, onde o autor descreve minuciosamente a formação de toda uma população de esmectitas de composição química veriável, com o aumento do grau de alteração do material. Sobre o minério oxidado, constam da literatura somente dois artigos, ambos relativos à laterita niquelífera de Barro Alto (KUHNEL et al., 1978; SCHELLMANN, 1983).

Resta, portanto, muito a ser feito com relação ao estudo dos nossos depósitos de níquel laterítico. Se é verdade que em linhas gerais os mais importantes estão bem caracterizados no que diz respeito à especificidade do perfil que apresentam e à organização espacial das zonas mineralizadas, muito pouco ainda se sabe em relação às características cristaloquímicas das fases portadoras de níquel.

\section{REFERÊNCIAS BIBLIOGRÁFICAS}

AMMOU-CHOKROUM, M. - 1969 - Contribution à l'étude de la distribution du nickel et des ferralites de Nouvelle-Calédonie. Compte Rendus, Paris, Academie Sciences, Séction D, 268:1563-1566.

- 1972 - Contribution à la valorisation des ferralites nickelifères de NouvelleCalédonie: distribution minéralogique des élements et étude de leur comportement au cours de la réduction solide-gaz des materiaux. Nancy, France, Université, 170p. (Thèse de Doctorat.)

ANGELI, N. - 1989 - Pesquisa dos jazimentos de níquel e geologia da Folha IpanemaMG. São Paulo, Instituto de Geociências, USP. 290p. (Tese de Doutoramento.) ; OLIVEIRA, S.M.B.; CHOUDHURI, A.; RUEDA, J.R.J. - 1984 - Avaliação preliminar dos produtos de alteração das rochas ultramáficas de Ipanema-MG. IN:
CONGRESSO BRASILEIRO DE GEOLOGIA, 33․, Rio de Janeiro, 1984. Anais. Rio de Janeiro, Sociedade Brasileira de Geologia.v.6, p.4.229-4.240.

AVIAS, J. - 1978 - L'évolution des idées et des connaissances sur la genèse et sur la nature des minerais de nickel, en particulier latéritiques, de leur découverte à nos jours. Bulletin BRGM, Paris, Séction II, (3):162-165.

BARBOUR, A.P. - 1978 - Maciço ultramáfico Ni-laterítico de Santa Fé, Goiás, Brasil. IN: CONGRESSO GEOLÓGICO ARGENTINO, 7º, Neuquem, 1978. Actas. v.1, p.119-131.

\& HYPÓLITO, R. - 1983 - Geological aspects of lateritisation and superficial geology of a mafic-ultramafic alkaline complex in Central Brasil. IN: PROCEEDINGS OF THE INTERNATIONAL SEMINAR ON LATERITISATION PROCESSES, 2 \%, São Paulo, 1983. São Paulo, IGCP/IAGC. p.137-146.

BERBERT, C.O. - 1977 - Rochas básicoultrabásicas no Brasil e suas potencialidades econômicas. IN: SEMINÁRIO DE ESTUDOS GEOLÓGICOS DO PARANÁ, 2, Curitiba, 1977. Curitiba, PR., CEGEP. p.1-51.

BERNARDELLI, A.L.; MELFI, A.J.; OLIVEIRA, S.M.B.; TRESCASES, J.J. 1983 - The Carajás nickel deposit. IN: PROCEEDINGS OF THE INTERNATIONAL SEMINAR ON LATERITISATION PROCESSES, 2․, São Paulo, 1983. São Paulo, IGCP/IAGC. p.107-118.

BIBENT, B. - 1972 - Sur l'altération experimentale par l'eau pure de deux roches ultrabasiques de Nouvelle-Calédonie: conséquences pour la formation des complexes latéritiques. Compte Rendus, Paris, Academie Sciences, Séction D, 274:1609-1612.

\& BOURGEOIS, A. - 1973-Resultats préliminaires d'une série d'expériences d'extraction du fer, du magnesium et du nickel de roches et mineraux nickelifères; leurs relations avec les caractéristiques minéralogiques des échantillons. Compte Rendus, Paris, Academie Sciences, Séction D, 276: 1929-1932.

BISH, O.L. \& BRINDLEY, G.W. - $1978-$ Deweylites, mixtures of poorly crystalline hydrous serpentine and talc-like minerals. Mining Magazine, London, 42:75-79.

BLOT, A.; CARN, N.; LEPRUN, J.C.; PION, J.C. - 1976 - Premier bilan des études géologiques et pédologiques d'un corps ultrabasique et de son contexte: Koussane, Se- 
negal Oriental. Cahiers ORSTOM, Paris, Série Géologie, 8:113-145.

BONIFAS, M. - 1959 - Contribution à l'étude géochimique de l'alteration lateritique. Mem. Serv. Geol. Als. - Lorr., v.17. $159 \mathrm{p}$.

BOSIO, N.J.; HURST, V.J.; SMITH, R.L. 1975 - Nickeliferous nontronite, a 15A garnierite, at Niquelândia, Goiás, Brazil. Clays and Clay Minerals, Lawrence, Kansas, 23(6):400-403.

BRINDLEY, G.W. \& HANG, P.T. - $1973-$ The nature of garnierites I. Clay Minerals, London, 21(1):17-40.

\& MAKSIMOVIC, Z. -1974 - The nature and nomenclature of hydrous nickelcontaining silicates. Clay Minerals, London, 10(2):271-277.

\& SOUZA, J.V. - 1975a - A goldencolored, ferri-nickel chloritic mineral from Morro do Níquel, Minas Gerais, Brazil. Clays and Clay Minerals, Lawrence, Kansas, 23(1):11-15.

$\&$

containing montmorillonites and chlorites from Brazil, with remarks on schuchardite. Mining Magazine, London, 40(1):141-152. -1978 - The structure and chemistry of hydrous nickel-containing silicate and aluminate minerals. Bulletin BRGM, Paris, Séction II, (3):233-245. ; BISH, D.L.; WAN, H.M. -1979 - Compositions, structures, and properties of nickel-containing minerals in the kerolitepimelite series. American Mineralogist, Washington, 64(5/6):615-625.

CERVELLE, B.D. \& MAQUET, M. - 1982 - Cristallochimie des lizardites substitueés $\mathrm{Mg}-\mathrm{Fe}-\mathrm{Ni}$ par spectrométrie visible et infrarouge proche. Clay Minerals, London, 17(3):377-392.

CHETELÂT, E.-1947 - Genèse et évolution des gites de nickel de Nouvelle-Calédonie. Bulletin de la Sociéte Géologique de France, Paris, 5(17): 105-160

CHUKROV, F.V.; GORSHOV, A.I.; SIVTSOV, A.V.; BARESOVSKAYA, V.V. 1983 - On the manganese mineralogy, in the lateritic weathering crusts of ultrabasic rocks. IN: PROCEEDINGS OF INTERNATIONAL THE SEMINAR ON LATERITISATION PROCESSES, 2․, São Paulo, 1983. São Paulo, IGCP/IAGC. p.147-158.

COLIN, F. - 1985 - Étude pétrologique des alterations de pyroxénite du gisement nickelifère de Niquelândia (Brésil). Paris, TDM ORSTOM. 137p.

; NOACK, Y.; TRESCASES, J.J.; NAHON, D. - 1985 - L'altération latéri- tique débutante des pyroxénites de Jacuba, Niquelândia, Brésil. Clay Minerals, London, 20(1):93-113.

COMBES, P.J. - 1963 - À propos du nickel dans les latérites nickelifêres de NouvelleCalédonie. Compte Rendus, Paris, Academie Sciences, Séction D, 256:211-212.

CORREA, S.L.A.; OLIVEIRA, N.P.; SCHWAB, R.G. - 1984 - Alguns aspectos mineralógicos e geoquímicos da laterita niquelífera do Vermelho, Serra dos Carajás, e suas implicações genéticas. IN: CONGRESSO BRASILEIRO DE GEOLOGIA, 33․, Rio de Janeiro, 1984. Anais. Rio de Janeiro, Sociedade Brasileira de Geologia, v. 10, p.4838-4849.

COSTA, J.L.G. -1970 - Nickel deposits of the São João do Tocantins massif: notes on economic geology and on exploration by C.N.T. IN: CONGRESSO BRASILEIRO DE GEOLOGIA, 24ㅇ, Brasília, 1970. Boletim de Resumos. Brasília, Sociedade Brasileira de Geologia. p.142-143.

COUTY, R. \& PERRUCHOT, A. - $1980-$ Étude par spectroscopie infrarouge de quelques gels silico-magnésiens. Compte Rendus, Paris, Academie Sciences, Séction D, 290:551-553.

COY-YLL, R. \& GUILLOUX, L. - $1972-$ Géochimie des élements de transition lors de processus d'altération de l'olivine. IN: PROCEEDINGS OF THE INTERNATIONAL GEOLOGICAL CONGRESS, 24\%, Montreal, 1972. v.10, p.112-120.

CRUZ, F.F.; BRENNER, T.L.; MOREIRA, A.F.S.; CUNHA, C.A.B.R.; GALLO, C.B.M.; FRANKE, N.D.; PIMENTEL, R.C. - 1986 - Jazida de Ni-Cu-Co de Fortaleza de Minas, MG. IN: PRINCIPAIS DEPÓSITOS MINERAIS DO BRASIL. Brasília, DNPM/CVRD. v.2, p.275-306.

DELMAS, A.B. - 1972 - Sur les rôles respectifs de la température et du débit au cours de l'altération expérimentale de I'olivine par lessivage à l'eau. Compte Rendus, Paris, Academie Sciences, Séction D, 274:2413-2415.

DEPARTAMENTO NACIONAL DA PRODUÇÃO MINERAL - DNPM - 1988 Anuário Mineral Brasileiro. Brasília, MME/DNPM. 351p.

DEYOUNG JR., J.H.; SUTPHIN, D.M.; WERNER, A.B.T; FOOSE, M.P. - 1985 - International strategic minerals inventory: Sumary Report - Nickel. Denver, CO., United States Geological Survey. 62p. (Circular, 930-D).

ESSON, J. \& SANTOS, L.C.S. - 1978a Chemistry and mineralogy of a section 
through lateritic nickel deposit at Liberdade, Brazil. Transactions of the Institution of Mining and Metallurgy, London, Section B, 87:53-60.

\& $-1978 \mathrm{~b}-$ The ocurrence, mineralogy, and chemistry of some garnierites from Brazil. Bulletin BRGM, Paris, Séction II, (3):263-274.

FELICÍSSIMO JR., J. - 1968 - Distritos ultrabásicos-alcálicos da bacia tectônica do Baixo Ribeira e seus aspectos econômicos, SP. Boletim SICEG, Ouro Preto, 6:90-134.

FERRAN, A. - 1974 - Panorama do níquel no Brasil. Geologia e Metalurgia, São Paulo, (35): 103-124.

GÉRARD, P. \& HERBILLON, A.J. - 1983 Infrared studies of Nibearing clay minerals of the kerolite-pimelite series. Clays and Clay Minerals, Lawrence, Kansas, 31(2):143-151.

GERTH, J. - 1990 - Unit-cell dimensions of pure and trace metal associated goethites. Geochimica and Cosmochimica Acta, London, 54(2):363-371.

GOLIGHTLY, J.P. - 1981 - Nickeliferous laterite deposits. Economic Geology, New Haven, $75^{\text {tn }}$ Anniversary Volume. p.710-735.

GONI, J. \& GUILLEMIN, C. - 1964 - Sur la localisation des éléments en trace dans les mineraux et les roches. Bullettin de la Société Française de Minéralogie et Cristallographie, Paris, 87(2):149-156.

HANG, P.T. \& BRINDLEY, G.W. - $1973-$ The nature of garnierites III. Clays And Clay Minerals, Lawrence, Kansas, 21(1):51-57.

JURKOVIC, I. - 1963 - Some geochemical aspects about the genesis of the nickel deposit Loma de Hierro (Venezuela). Geoloski Vjesnik, Zaghreb, 17(1):103-112.

KUHNEL, R.A.; ROORDA, H.J.; STEENSMA, J.J. - 1978 - Distribution and partitioning of elements in nickeliferous laterites. Bulletin BRGM, Paris, Séction II, 3:191-206.

LACROIX, A. - 1942 - Les peridotites de la Nouvelle-Calédonie, leurs serpentines et leurs gites de nickel et de cobalt: les gabbros qui les accompagenent. Paris, Academie de Scienses 143p. (Memoir, 66).

LAGES, S.M.; ANDRADE, M.R.; OLIVAS, L.A. - 1976 - Economia mineral do níquel. Rel. DNPM para o XXIX Congr. Bras. Geol. 32p.

LANGER, E. - 1969 - Die Nickẹlelagertatten des Morro do Niquel in Minas Gerais, Brasilien: Ihr Aufschuss, ihre Bemusterung und Bewertung. Clausthaler Hefte zur La- gerstattenkunde und Geochemie des Mines, Stuttgart, Heft 8. 64p.

LELONG, F.; TARDY, Y; GRANDIN, G.; TRESCASES; J.J.; BOULANGÉ, B. 1976 - Pedogenesis, chemical weathering, and processes of formation of some supergene ore deposits. IN: HANDBOOK OF STRATABOUND AND STRATIFORM ORE DEPOSITS. K.H. Wolf, ed. Amsterdan, Elsevier. v.3, p.93-173.

MAKSIMOVIC, Z. - 1966 - B-kerolitepimelite series from Goles Mountain, Yougoslavia. IN: PROCEEDINGS OF THE INTERNATIONAL CLAY CONFERENCE, Jerusalem, 1966. p.97-105. - 1972 - Nimesite: a new septochlorite from a bauxite deposit near Megara (Greece). Bulletin Science Yugoslavia. p. 224-226.

- 1975 - The isomorfous series lizardite-nepouite. Int. Geol. Review, 17(9):1035-1040.

MANCEAU, A. - 1984 - Localisation du nickel dans les phylossilicates: aplications aux mineraux de nickel de Nouvelle-Calédonie. Paris, Université. 99p. (Thèse de Doctorat). \& CALAS, G. - 1983 - Cristallochemistry of secondary nickeliferous minerals resulting from the alteration of new-caledonian peridotites. Science Géologique Memoire, Strasbourg, 73:153-159.

; LLORCA, S.; CALAS, G - 1987 -

Cristal chemistry of $\mathrm{Co}$ and $\mathrm{Ni}$ in lithiophorite and asbolane from New Caledonia. Geochimica and Cosmochimica Acta, London, 51(1):105-113.

MAQUET, M.; CERVELLE, B.D.; GOUET, G. -1981 - Signatures of $\mathrm{Ni}^{2}$ and $\mathrm{Fe}^{3}$ in the optical spectra of limonitic ore from New Caledonia: application to the determination of the nickel content. Mineralium Deposita, Berlin, 16(4):357-373.

MELFI, A.J. - 1974 - Características geoquímicas e mineralógicas dos estádios iniciais de alteração superficial das rochas ultrabásicas de Barro Alto (GO). Boletim IGUSP, São Paulo, 5:117-128.

;TRESCASES, J.J.; OLIVEIRA, S.M.B. - 1980 - Les laterites nickelifères du Brésil. Cahiers ORSTOM, Paris, Série Géologie, 11(1):15-42.

;CARVALHO, A; OLIVEIRA, S.M.B.; RIBEIRO FILHO, E.; FORMOSO, M.L. - 1988 - The lateritic ore deposits of Brazil. Science Géologique Bulletin, Paris, 41:5-36.

MERCADO, J.M. - 1981 - Geochemistry of the laterites in Nonoc Islands, Surigao Province, Phillipines. IN: PROCEEDINGS OF 
THE INTERNATIONAL SEMINAR OF LATERITISATION PROCESSES, $1^{\circ}$, Trivandrum, 1981. p. 45-57.

NAHON, D.; PAQUET, H.; DELVIGNE, J. -1982 - Lateritic weathering of ultramafic rocks and the concentration of nickel in the western Ivory Coast. Economic Geology, New Haven, 77(8):1159-1175.

NILSON A. A.; SANTOS, M.M.; CUBA, E.A.; SÁ, C.M.G. - 1986 - Jazida de níquel, cobre e cobalto de Americano do Brasil, GO. IN: PRINCIPAIS DEPÓSITOS MINERAIS DO BRASIL. Brasília, DNPM/CVRD. v.2, p. 257-273.

OGURA, Y. - 1977 - Mineralogical studies on the occurrence of nickeliferous laterite deposits in the Southwestern Pacific Area. Mines and Geology Bulletin, Alaska, 27(3): 379-399.

OLIVEIRA, S.M.B. - 1980a - Transformações mineralógicas relacionadas à meteorização de dunitos serpentinizados. IN: CONGRESSO BRASILEIRO DE GEOLOGIA, 31․, Camboriú, SC, 1980. Anais. Camboriú, SC, Sociedade Brasileira de Geologia, v. 4, p. 2182-2192.

- 1980b - Alteração intempérica das rochas ultrabásicas de Santa Fé (GO) e gênese do depósito niquelífero. São Paulo, Instituto de Geociências, USP. 216p. (Tese de Doutoramento).

\& TRESCASES, J.J. - $1980-$ Geo-

química da alteração supérgena das rochas ultramáficas de Santa Fé (Goiás-Brasil). Revista Brasileira de Geociências, São Paulo, 10(4):243-257.

\& _ - 1982 - Estudo minera-

lógico e geoquímico da laterita niquelífera de Niquelândia-GO. IN: CONGRESSO BRASILEIRO DE GEOLOGIA, 32\%, Salvador, 1982. Anais. Salvador, Sociedade Brasileira de Geologia. v.3, p. 1183-1190. \& -1985 - O depósito de níquel de Jacupiranga (SP): evolução mineralógica e geoquímica. Revista Brasileira de Geociências, São Paulo, 15(3):249-254.

; MELFI, A.J.; CARVALHO, A.;

FRIEDRICH, G.: MARKER, A.; KANIG, M. - 1988 - Lateritic evolution of the Jacupiranga alkaline complex, SP. Geochimica Brasiliensis, Rio de Janeiro, 2 (2): 119-126.

- 1990 - Os depósitos de níquel laterítico do Brasil. São Paulo, Instituto de Geociências, USP, 89p. (Trabalho de LivreDocência).

PECORA, W.T. - 1944 - Nickel silicate and associated nickel-cobalt-manganese oxide deposits near São José dos Tocantins, Goiás,
Brazil. Washington, United States Geological Survey. 59p. (Bulletin, 935-E).

PEDRO, G. \& BITTAR, K.E. - 1966 - Contribution à l'étude de la genèse des sols hypermagnésiens: recherches expérimentales sur l'altération chimique des roches ultrabasiques (serpentinites), Annales Agronomiques, Paris, 17(6):611-651.

\& DELMAS, A.B. - 1971 - Sur l'altération expérimentale de l'olivine par lessivage à l'eau et la mise en evidence de trois grands domaines d'évolution géochimique. Compte Rendus, Academie Sciences, Paris, Séction D, 273:1543-1546.

PELLETIER, B - 1983 - Localisation du nickel dans les mineraux "garnieritiques" de Nouvelle-Calédonie. Sciences Géologiques Memoire, Strasbourg, 73:173-183.

PERCIVAL, F.G. - 1965 - The lateritic iron deposits of Conakry. Transactions of the Institution of Mining and Metallurgy, London, 74(8):429-462.

PERRUCHOT, A - 1976a - Contribution à l'étude de la formation de gites silicatés de nickel. Bulletin de la Société Française de Minéralogie et Cristallographie, Paris, 99(2):225-233.

- 1976b - Contribution à l'étude d'échange d'ions dans les gels silicatés. Bulletin de la Société Française de Minéralogie et Cristallographie, Paris, 99(2):234-242.

PONCELET, G.; JACOBS, P.; DELANNAY, F.; GENET, M.; GÉRARD, P.; HERBILLON, A. - 1979 - Étude préliminaire sur la localisation du nickel dans une garnierite naturelle. Bulletin de Minéralogie, Paris, 102(4):379-385.

SAHOO, R.K.; KAADEN, G.V.D.; MULLER, G. - 1981 - The mineralogy and geochemistry of the nickeliferous laterite of Sukinda, Orissa, India. IN: PROCEEDINGS OF THE INTERNATIONAL SEMINAR OF LATERITISATION PROCESSES, $1^{\circ}$, Trivandrun, 1981. p.77-85.

SANTOS, J.F. - 1974 - Fatores de controle na concentração de níquel laterítico condicionado pela evolução geológica e geomorfológica do complexo básico-ultrabásico de São João do Piauí (PI). IN: CONGRESSO BRASILEIRO DE GEOLOGIA, 28\%, Porto Alegre, 1974. Anais. Porto Alegre, Sociedade Brasileira de Geologia. v.6, p.25-32.

SANTOS-YNIGO, L. - 1964 - Distribution of iron, alumina and silica in the Pujada Laterite of Mati, Davao province, Mindanao Island (Phillipines). IN: PROCEEDINGS 
OF THE INTERNATIONAL GEOLOGICAL CONGRESS, 22\%, New Delhi, 1964. v.14, p.126-141.

SCHELLMANN, W. - 1978 - Behavior of nickel, cobalt and chromium in ferruginous lateritic nickel ores. Bulletin BRGM, Paris, Séction II, (3):275-282.

- 1983 - Geochemical principles of lateritic nickel ore formation. IN: PROCEEDINGS OF THE INTERNATIONAL SEMINAR OF LATERITISATION PROCESSES, 2․, São Paulo, 1983. p.119-135.

- 1989 - Composition and origin of lateritic nickel ore at Tagaung Taung, Burma. Mineralium Deposita, Berlin, 24(3):161-168.

SCHOBBENHAUS, C.; CAMPOS, D.A ; DERZE, G.R.; ASMUS, H.E. - $1984-$ Geologia do Brasil. Brasília, DNPM. 501p.

SCHWERTMANN, U. \& LATHAM, M. 1986 - Properties of iron oxides in some New Caledonian oxisols. Geoderma, 39(2): 105-123.

; GASSER, U.; STICHER, H. 1989 - Chromium-for-iron substitution in synthetic goethites. Geochimica and Cosmochimica Acta, London, 53(11):1293-1297.

SILVA, A.C.G.A. - 1980 - Mineralogia, material amorfo, evolução genética e estudo do $\mathrm{Ni}$ e $\mathrm{Cr}$ no perfil de alteração do maciço ultramáfico de Santa Fé-GO. São Paulo, Instituto de Geociências, USP. 136p. (Dissertação de Mestrado).

SOUZA, J.V.; SANTOS, P.; SANTOS, H. 1978 - Caracterização mineralógica de algumas argilas niquelíferas brasileiras. Cerâmica, São Paulo, 24(108):434-446.

SPRINGER, G. - 1974 - Compositional and structural variation in garnierites. Canadian Mineralogist, Toronto, 12(3):381-388.

ST ${ }^{\wedge}$ CHE, G.A. - 1974 - Untersuchen zur Geologie, Petrographie, Metamorphose und Genese de basich-ultrabasichen Massivs von Barro Alto, Goiás (Brasilien). Clausthal, Technische Universitat. 198p. (Tese de Doutoramento).

STIERS, W. \& SCHWERTMANN, U. - 1985 - Evidence for manganese substitution in synthetic goethite. Geochimica and Cosmochimica Acta, London, 49(12):1909-1911.

STROESSEL, R.R. $-1988-25^{\circ} \mathrm{C}$ e 1 atm dissolution experiments of sepiolite and kerolite. Geochimica and Cosmochimica Acta London, 52(3):365-374.
TRESCASES, J.J. - 1975 - L'évolution supergène des roches ultrabasiques en zone tropicale. Paris, ORSTOM. 259p. (Mémoire, 78).

\& OLIVEIRA, S.M.B. $-1978-$ Alteração dos serpentinitos de Morro do Níquel (MG). IN: CONGRESSO BRASILEIRO DE GEOLOGIA, 30\%, Recife, 1978. Anais. Recife, PE., Sociedade Brasileira de Geologia. v.4, p.1655-1669.

\& $\quad-$ A jazida de níquel de Barro Alto. IN: SIMPÓSIO DE GEOLOGIA DO CENTRO-OESTE, 1․, Goiânia, 1981. Atas. Goiânia, Sociedade Brasileira de Geologia. p.519-538.

- 1986 - Nickeliferous laterites: a review on the contribution of the last ten years. Geol. Surv. India Mem., 120:51-62. ; DINO, R.; OLIVEIRA; S.M.B. 1986 - Un gisement de nickel supèrgene en zone semi-aride: São João do Piauí (Brésil). IN: GEOCHEMISTRY AND MINERAL FORMATION IN THE EARTH SURFACE. R.R. Clemente \& Y. Tardy, ed. Madrid, p.273-288.

TROLY, G.; ESTERLE, M.; PELLETIER, B; REIBELL, W. - 1979 - Nickel deposits in New Caledonia: some factors influencing their formation. IN: PROCEEDINGS OF THE INTERNATIONAL SYMPOSIUM OF LATERITISATION PROCESSES, New Orleans, 1979. p.81-119.

UYEDA, N.; HANG P.T; BRINDLEY, G.W. - 1973 - The nature of garnierites III. Clays and Clay Minerals, Lawrence, Kansas, 21(1):41-50.

VELDE, B. - 1988 - Experimental pseudomorphism of diopside by talc and serpentine in $(\mathrm{Ni}, \mathrm{Mg}) \mathrm{Cl}_{2}$ aqueous solutions. Geochimica and Cosmochimica Acta, London, 52(4):415-424.

VLETTER, D.R. - 1955 - How cuban ore was formed: a lesson in laterite genesis. Engeenering and Mining Journal, New York, 156(10):84-87.

WIEWIORA, A. \& SZPILA, K. - $1975-\mathrm{Ni}-$ ckel containing regularly interstratified chlorite-saponite from Szklary, Lower Silesia, Poland. Clay Minerals, London, 23(1):91-96.

ZEISSINK, H.E. - 1969 - The mineralogy and geochemistry of a nickeliferous laterite profile (Greenvale, Queensland, Australia). Mineralium Deposita, Berlin, 4(2):132-152.

Endereço do autor:

Sonia Maria Barros de Oliveira - Universidade de São Paulo - Instituto de Geociências - C.P. 20899 - 01498 - São Paulo (Brasil). 